\title{
Prevalence and prognosis of cerebrovascular accidents and its subtypes: $A$ cross-sectional study in Hail region, Saudi Arabia.
}

\author{
Nawaf $A^{1^{*}}$, Hala $A^{1}$, Ahmed $A^{1}$, Areeb $A^{1}$, Latifah $A^{1}$, Abdullah $A^{1}$, Khalid $A^{1}$, Turki $A^{1}$, Omar $A^{1}$, \\ Shuaa $A^{1}$, Sasikumar $D^{2}$ \\ ${ }^{1}$ Department of Internal Medicine, College of Medicine, University of Hail, Saudi Arabia \\ ${ }^{2}$ Department of Biochemistry, College of Medicine, University of Hail, Saudi Arabia
}

\begin{abstract}
Cerebrovascular accidents or stroke is an important healthcare concern. Ischemic stroke is an acute neurologic deficit that results from focal cerebral ischemia leading to permanent tissue damage (infarction). The cause is commonly associated with atherothrombotic occlusion of large arteries, cerebral embolism (embolic infarction), non-thrombotic occlusion of small, deep cerebral arteries (lacunar infarction), and proximal arterial stenosis with hypotension that decreases cerebral blood flow in arterial watershed zones (hemodynamic stroke). Stroke is being observed as a rapidly growing problem and an important cause of illness and death in Saudi Arabia. The aims of this cross-sectional study were to establish the prevalence and prognosis of cerebrovascular accidents and its subtypes in Hail Region, Saudi Arabia. Data were collected on all patients who experienced CVAs between September 2017-January 2018, used the diagnosis coding program in King Khalid Hospital in Hail, Saudi Arabia. Out of 190 patients $63.7 \%$ were male and $36.3 \%$ were female. The overall incidence of ischemic stroke after acute myocardial infarction was $85.26 \%$. Hypertension, diabetes mellitus were shows as more risk factors for ischemic stroke type compared with hemorrhagic stroke, and embolic stroke. The overall status of stroke patients were shown $92.1 \%$ live and $7.9 \%$ were dead. Incidence of complicating hospitalizations with cerebrovascular accident continues to grow and is associated with increased mortality and adverse discharge. This highlights the need for early diagnosis, better risk stratification, and preparedness for need for complex long-term care in this vulnerable population.
\end{abstract}

Keywords: Cerebrovascular accidents, Ischemic stroke, Hemorrhagic stroke, Embolic stroke, Myocardial infarction. Abbreviations: CVA: Cerebrovascular Accident; SAH: Subarachnoid Hemorrhage

Accepted on January 30, 2019

\section{Introduction}

Stroke is a major cerebrovascular disease resulting in high mortality and persistent disability in adults across the world. Besides coronary heart disease and cancer, stroke is the commonest cause of death in most industrialized countries [1]. Cerebrovascular accidents (CVAs) are the fifth leading cause of mortality and the leading cause of serious, long-term disability in the United States. Most strokes (87\%) are ischemic, caused by thrombotic or embolic occlusion of a major artery [2]. CVA has traditionally been divided into infarcts (embolic or thrombotic) and hemorrhagic lesions based on the pathologic and radiographic appearance. Infarcts are further subdivided into lacunar and cerebral infarcts. Similarly, hemorrhagic lesions are subdivided into intracerebral hemorrhage and subarachnoid hemorrhage (SAH).

CVA or stroke occurs when blood flow to a part of the brain is prevented due to blockage, or rupture of blood vessels [3]. Recent statistics on European cardiovascular diseases indicate that stroke is responsible for 4 million deaths, with over 1.9 million deaths recorded in the European Union [4]. In 75\% of the cases, the stroke attack is attributed to blood clot, i.e. ischemic stroke. The remaining cases are attributed to ruptured blood vessel in the brain, i.e. hemorrhagic stroke. The established risk factors, including arterial hypertension, diabetes mellitus, cigarette smoking, micro-vascular rupture, hyperlipidemia, age and observed comorbidity such as sickle cell disease, human immunodeficiency virus/acquired immune deficiency syndrome infection and cerebral malaria are increasingly being encountered in the tropics [5].

Approximately $90 \%$ of CVAs were attributed to 10 risk factors that are preventable (hypertension, regular physical inactivity, high apolipoprotein B/A1 ratio, insufficient diet quality, increased waist/hip ratio, psychosocial factors, current smoking, cardiac causes, heavy alcohol consumption, and diabetes mellitus) [6]. Stroke is being observed as a rapidly growing problem and an important cause of illness and death in Saudi Arabia. Therefore, it becomes one of the most imperative social and economic medical issues in the Kingdom [7]. The aims of this cross-sectional study were to establish the prevalence and prognosis of cerebrovascular accidents and its subtypes in Hail Region, Saudi Arabia. 


\section{Materials and Methods}

\section{Study design, study setting and subjects}

This study was a cross-sectional study. Defining a CVA as an acute complication of certain chronic diseases with clinical manifestations requiring immediate medical care and hospitalization, we collected data on all patients who experienced CVAs between September 2017-January 2018, we used the diagnosis coding program in King Khalid Hospital in Hail, Saudi Arabia city to look for any patient who had CVA in the last five years, we found more than 300 patients there were diagnosed with CVA and had been admitted to the hospital for full assessment and management. The local institutional ethics committee, College of Medicine, University of Hail, Saudi Arabia, approved the research proposal and design (Approval No: EC-0022). We started contacting our patients to have their consent to open their medical records and we received 190 consent. We design a collector form to collect our data, we went through their medical history and some specific lab and radiological investigations. All the data obtained were transferred to an appropriate record form (Figure 1). No additional investigations were pursued with the patients' families or with physicians.

\section{Statistics analysis}

All the data from record form were entered in a system in the Microsoft Excel $^{\circledR}$ table format then the final file was exported to the Statistical Package for the Social Sciences V.23 (SPSS) for analysis. The age of patients were represented in mean and standard deviation. The categorical variables was expressed as total numbers and percentages. Frequency tables were used to present the distribution of nominal variables. Descriptive statistics were used to assess the baseline demographics; they were carried out by calculating the frequencies and percentages. Summaries for groups of cases were calculated and represented in the legacy dialogs bars with a level of $95 \%$ confidence intervals $(\mathrm{CI})$.

\section{Results}

Overall, 190 cerebrovascular accidents or stroke patients were reported and stratified by sex. Out of 190 patients $121(63.7 \%)$ were male and $69(36.3 \%)$ were female. The average age of patients were $63.68 \pm 15.82$ years, $62.66 \pm 15.99$ were male, and $65.48 \pm 15.47$ were female. Table 1 represents in total number of patients in the cross tabulation of gender with the stroke subtype. The ischemic stroke shows more in male compared with female. Comparatively male CVA patients are almost double compared with female CVA patients.

Figure 2 showed the nationality wise stroke subtypes of patients with CVA in Hail region. To compare with other nationality, $80.53 \%$ (47.37\% male; $33.16 \%$ female) of CVA patients are Saudi. We found $5.27 \%$ (both male and female) of CVA patients were Indians. But the other nationalities showed in less numbers compared with Saudi. Figure 3 represents the percentage of stroke subtypes of CVA patients in Hail region. The overall incidence of ischemic stroke within 5 months after acute myocardial infarction was $85.26 \%$ over the period September 2017 - January 2018. Ischemic stroke was more predominant

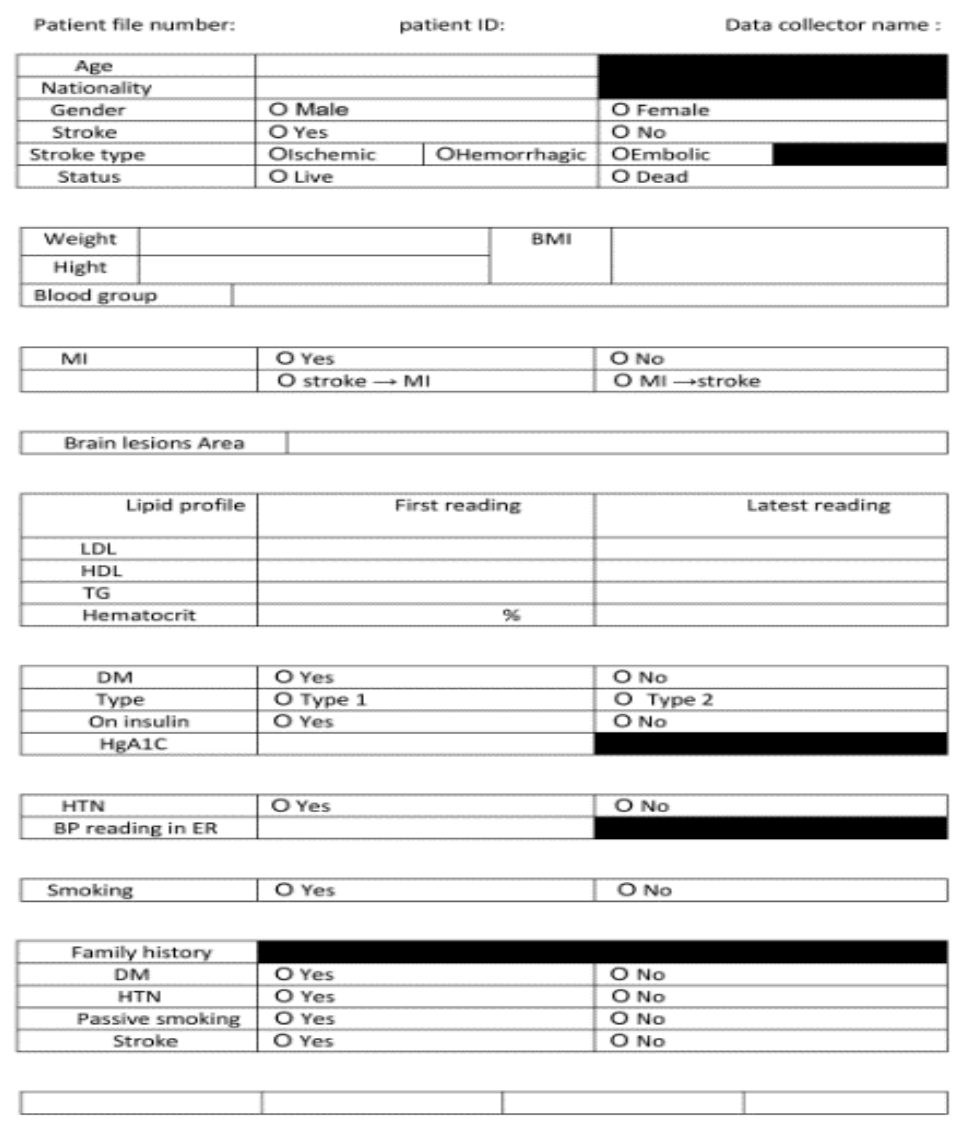

Figure 1. The model of record form which was filled the collected patient's data. 
Citation: Nawaf A, Hala A, Ahmed A, et al. Prevalence and prognosis of cerebrovascular accidents and its subtypes: A cross-sectional study in Hail region, Saudi Arabia. J Neurol Neurorehabil Res. 2019;4(1):1-8.

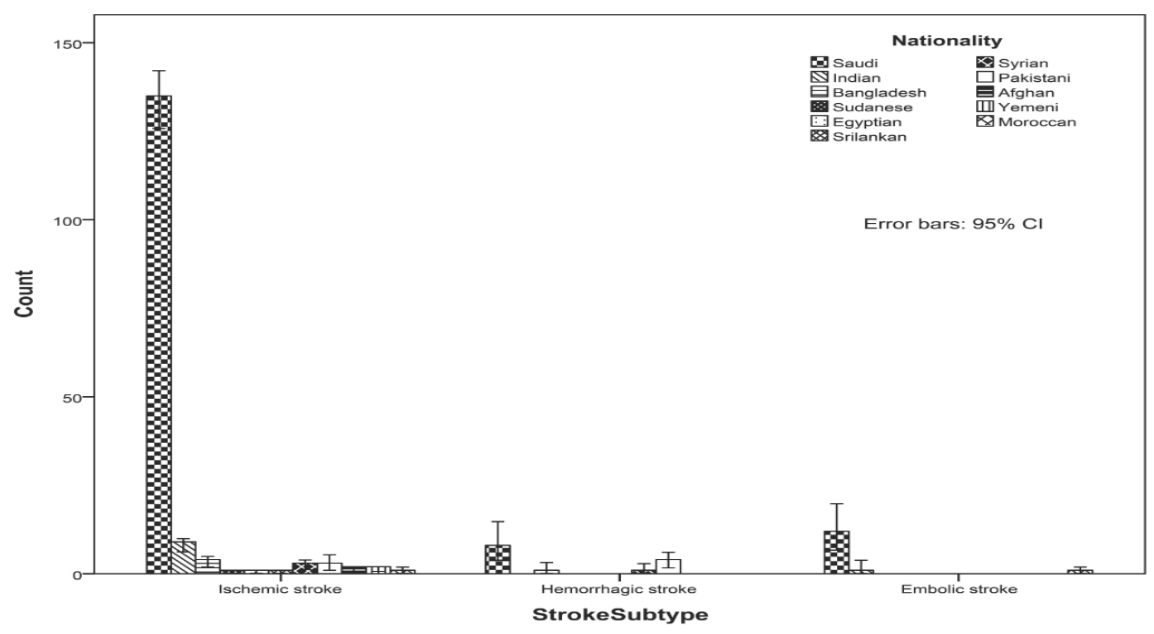

Figure 2. Shows the stroke subtypes of patients with their nationality in Hail region, Saudi Arabia.

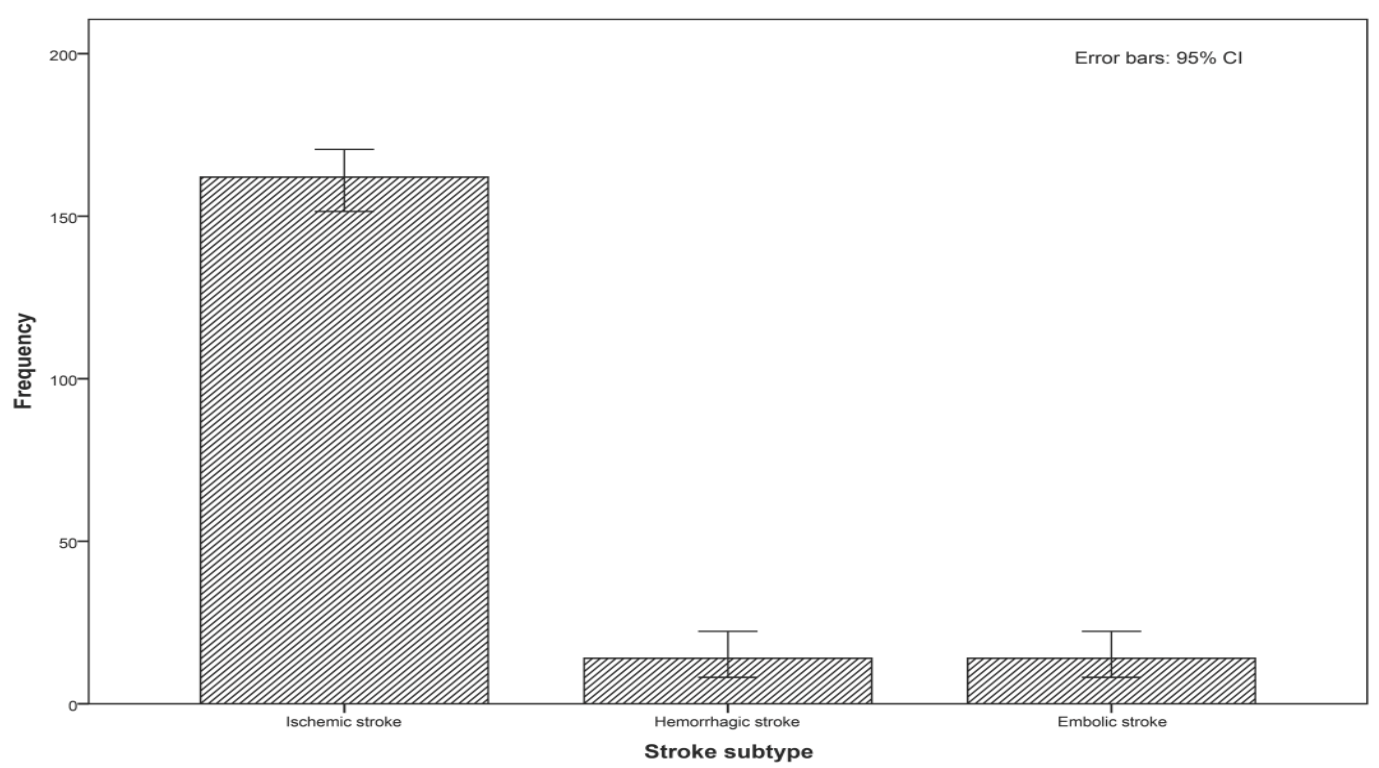

Figure 3. Represents the percentage of stroke subtypes in 190 patients with cerebrovascular accidents from Hail region between September 2017-January 2018.

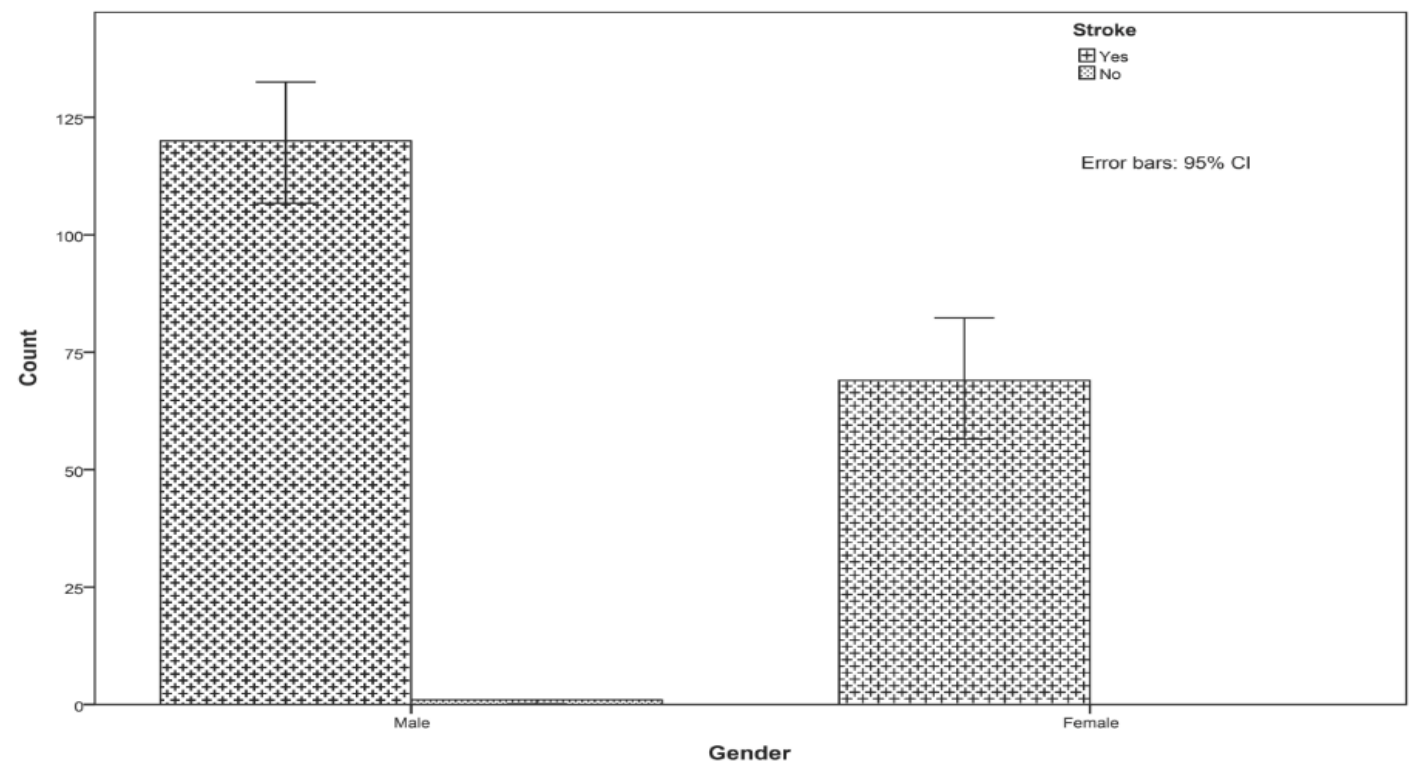

Figure 4. Prevalence of stroke among gender which shows $99.5 \%$ had stroke in cerebrovascular accidents patients. 
compared with other subtypes of CVA. But hemorrhagic stroke and embolic stroke were shown an equal percentage $(7.37 \%)$ of CVA patients.

The prevalence of stroke among gender were shown in Figure 4. Findings from this cross-sectional studies suggest male stroke patients were significantly increased compared with female. $99.5 \%$ of patients had stroke, of this $63.16 \%$ male and $36.32 \%$ female CVA patients. Figures 5-7 showed the prevalence of stroke subtypes with the established risk factors, including arterial hypertension, diabetes mellitus, and cigarette smoking. Hypertension, diabetes mellitus were shows as more risk factors for ischemic stroke type compared with hemorrhagic stroke, and embolic stroke. But cigarette smoking is not a major risk factors for ischemic stroke. From the legacy dialogs bars of stroke subtype in relation to hypertension patients showed $70.53 \%$ of ischemic stroke had hypertension but $14.74 \%$ showed ischemic stroke without hypertension. The other CVA subtypes like hemorrhagic stroke and embolic stroke had $6.32 \%$ and $6.84 \%$ of hypertension respectively. Its indicates only $1.05 \%$ of hemorrhagic stroke and $0.53 \%$ of embolic stroke patients had CVA without hypertension.
From the Figure 6 showed $63.68 \%$ of ischemic stroke, $5.26 \%$ of hemorrhagic stroke and $5.26 \%$ of embolic stroke patients were not smokers. This indicates smoking is not a major risk factors for stroke. Figure 7 indicates over all $67.37 \%$ of CVA patients (all subtypes) had diabetes mellitus which is indicates that the diabetes mellitus is a one of the major risk factor for CVA with stroke.

The association between myocardial infarction and stroke subtype were shown in Figures 8 and 9. Stroke with myocardial infarction were more predominate in ischemic stroke compared with other stroke subtypes. Myocardial infarction than stroke were significantly increased in embolic stroke compared with hemorrhagic stroke. The overall $74.74 \%$ of CVA patients showed with myocardial infarction, from this $68.09 \%$ of ischemic stroke patients, $23.40 \%$ of embolic stroke patients and $2.13 \%$ of hemorrhagic stroke patients had MI then stroke. It is indicates that MI also major risk factor for CVA patients.

Table 2 shows the cross tabulation on status of the CVA patients with their stroke subtype. $77.9 \%$ of patients with ischemic stroke were live but $7.4 \%$ of patients were dead. All the hemorrhagic

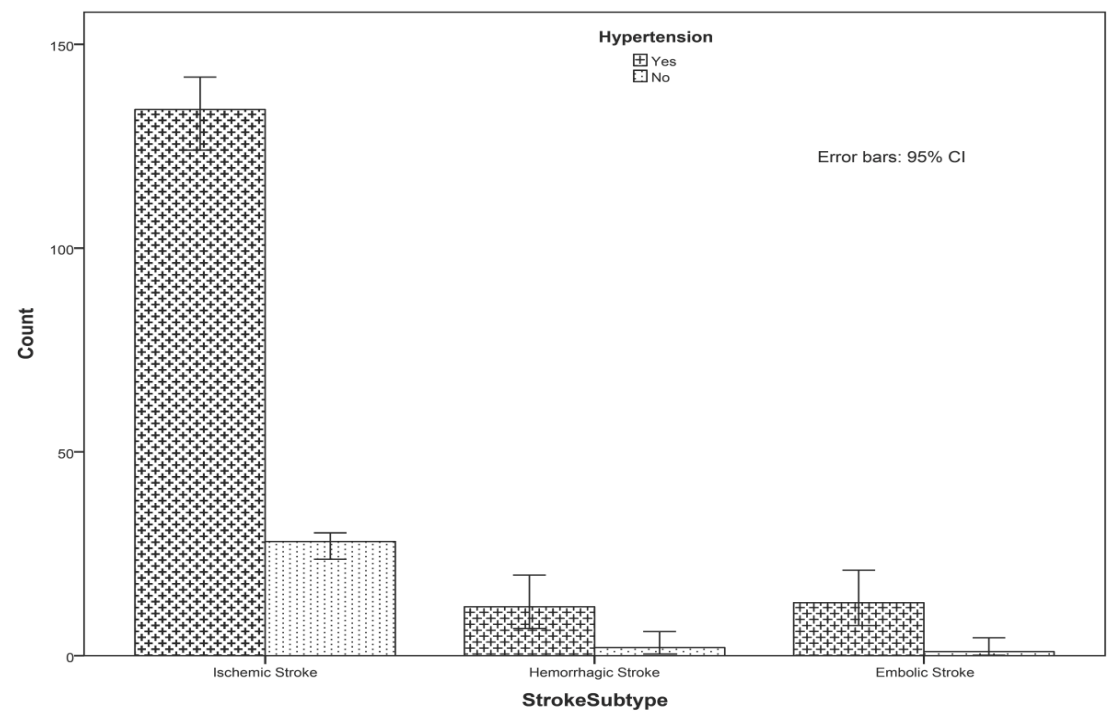

Figure 5. Prevalence of stroke subtype in relation to hypertension patients with cerebrovascular accidents from Hail region.

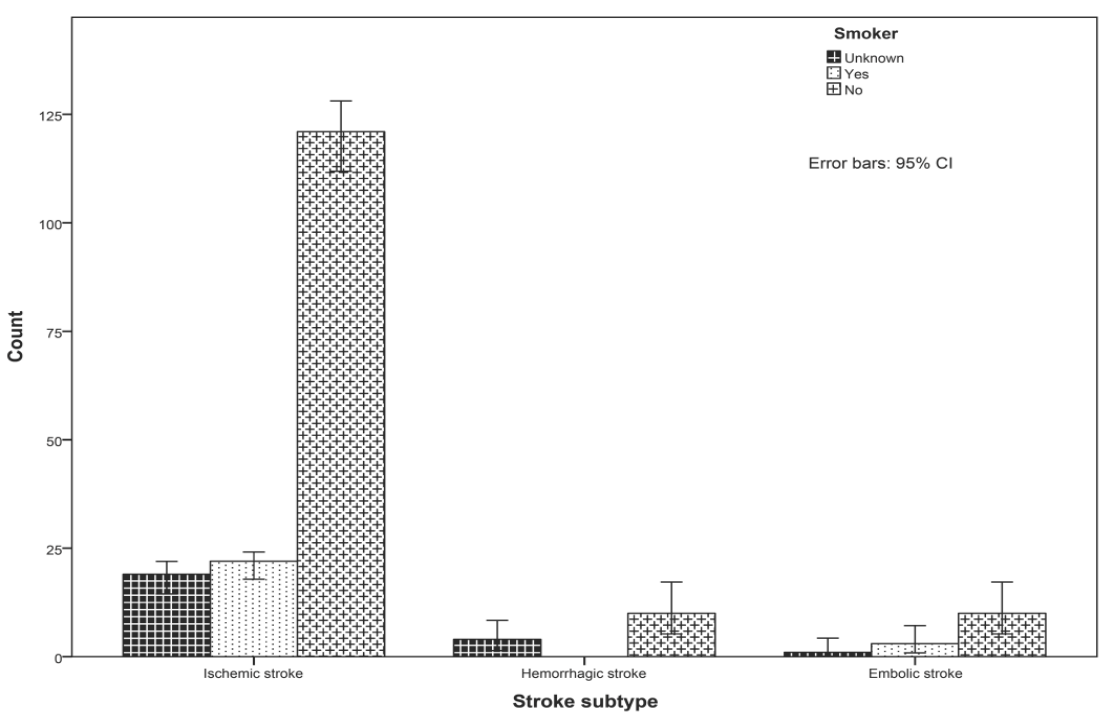

Figure 6. Prevalence of stroke subtype in relation to smokers hypertension patients with cerebrovascular accidents from Hail region in Saudi Arabia. 
Citation: Nawaf A, Hala A, Ahmed A, et al. Prevalence and prognosis of cerebrovascular accidents and its subtypes: A cross-sectional study in Hail region, Saudi Arabia. J Neurol Neurorehabil Res. 2019;4(1):1-8.

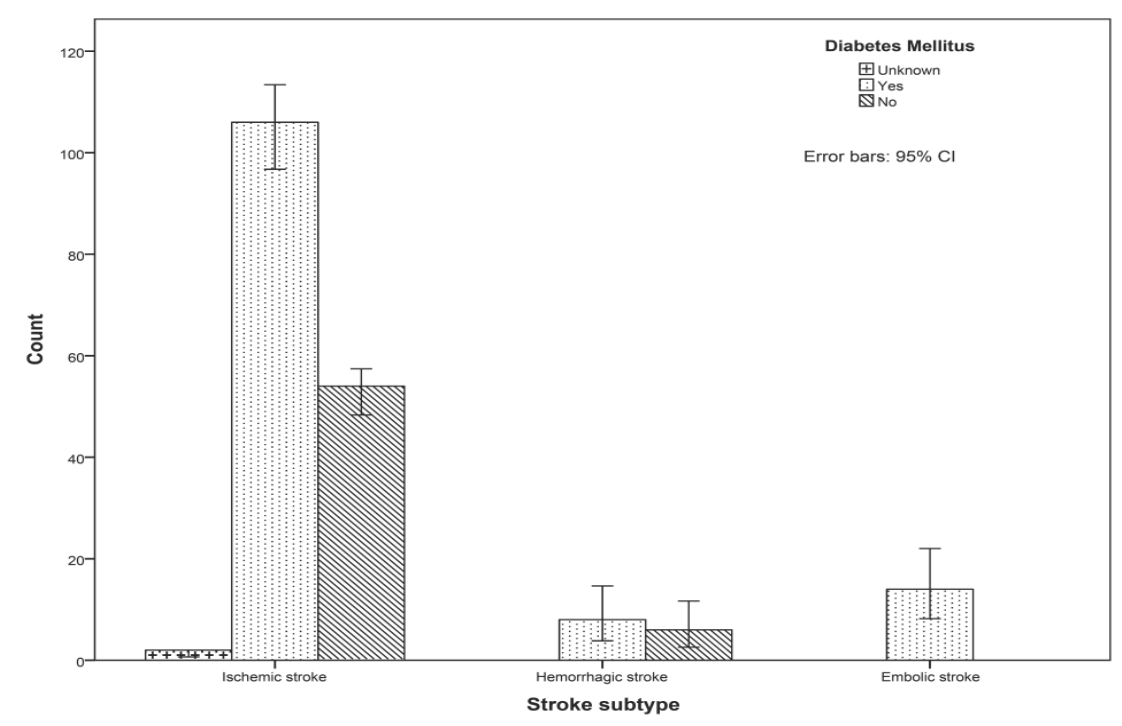

Figure 7. Association of diabetes mellitus with stroke subtype patients with cerebrovascular accidents from Hail region in Saudi Arabia. [55.79\%-ischemic stroke with DM; 4.21\% - hemorrhagic stroke with DM and 7.37\% - embolic stroke with DM].

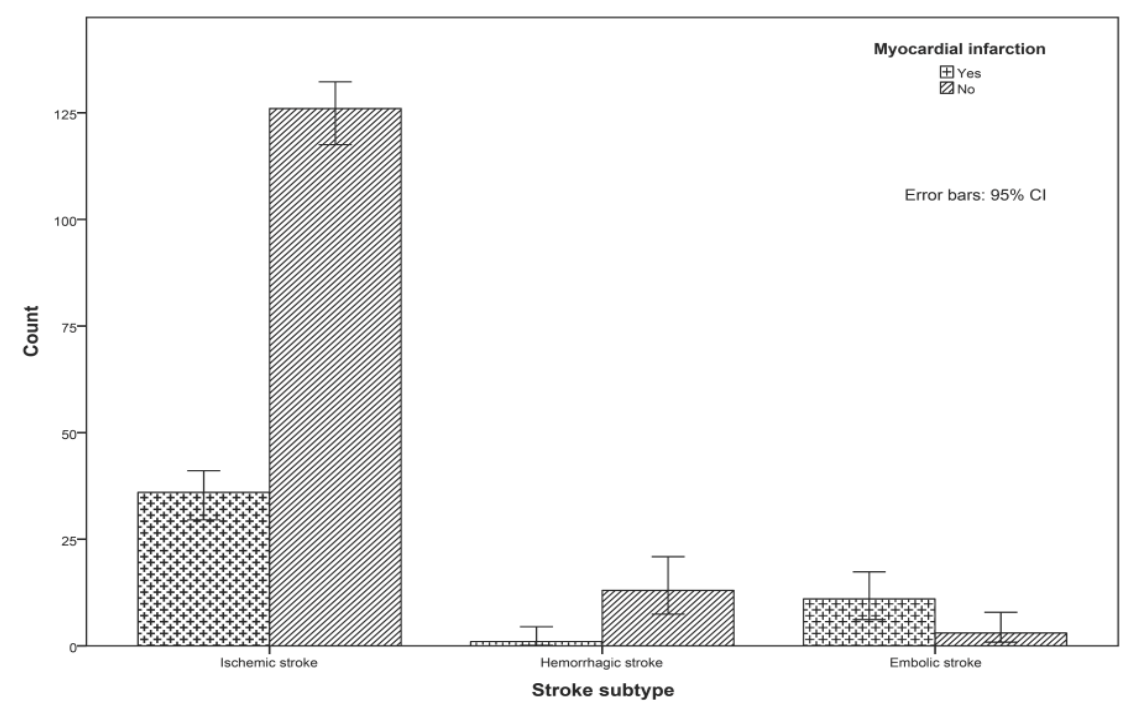

Figure 8. The prevalence and prognosis of stroke subtypes with myocardial infarction patients with cerebrovascular accidents from Hail region in Saudi Arabia. [18.95\% ischemic stroke with MI; 66.32\% ischemic stroke without MI; 0.53\% hemorrhagic stroke with MI; $6.84 \%$ hemorrhagic stroke without MI; 5.79\% embolic stroke with MI; 1.58\% embolic stroke without MI].

Table 1. Shows the cross tabulation of gender with the stroke subtype of patients in Hail region.

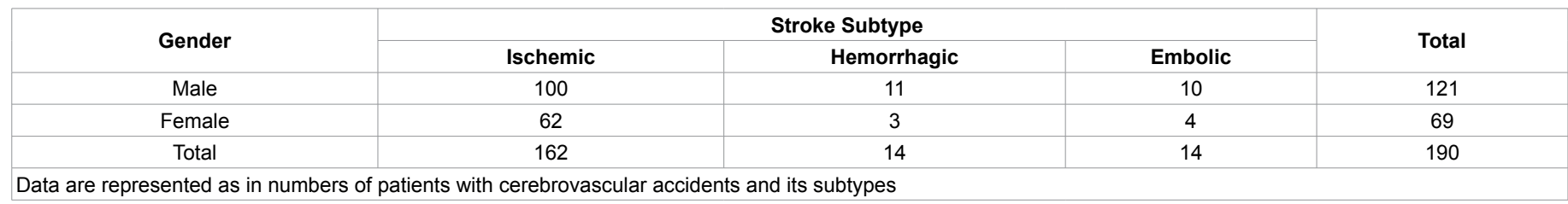

Table 2. Shows the cross tabulation on status of the CVA patients with their stroke subtype.

\begin{tabular}{|c|c|c|c|c|}
\hline \multirow{2}{*}{\multicolumn{3}{|c|}{ CVA Patients }} & \multicolumn{2}{|c|}{ Status } \\
\hline & & & Live & Dead \\
\hline \multirow{6}{*}{ 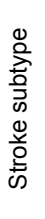 } & \multirow{2}{*}{ Ischemic stroke } & Count & 148 & 14 \\
\hline & & $\%$ of Total & $77.9 \%$ & $7.4 \%$ \\
\hline & \multirow{2}{*}{ Hemorrhagic stroke } & Count & 14 & 0 \\
\hline & & $\%$ of Total & $7.4 \%$ & $0.0 \%$ \\
\hline & \multirow{2}{*}{ Embolic stroke } & Count & 13 & 1 \\
\hline & & $\%$ of Total & $6.8 \%$ & $0.5 \%$ \\
\hline & \multirow{2}{*}{ Total } & Count & 175 & 15 \\
\hline & & $\%$ of Total & $92.1 \%$ & $7.9 \%$ \\
\hline
\end{tabular}


Citation: Nawaf A, Hala A, Ahmed A, et al. Prevalence and prognosis of cerebrovascular accidents and its subtypes: A cross-sectional study in Hail region, Saudi Arabia. J Neurol Neurorehabil Res. 2019;4(1):1-8.

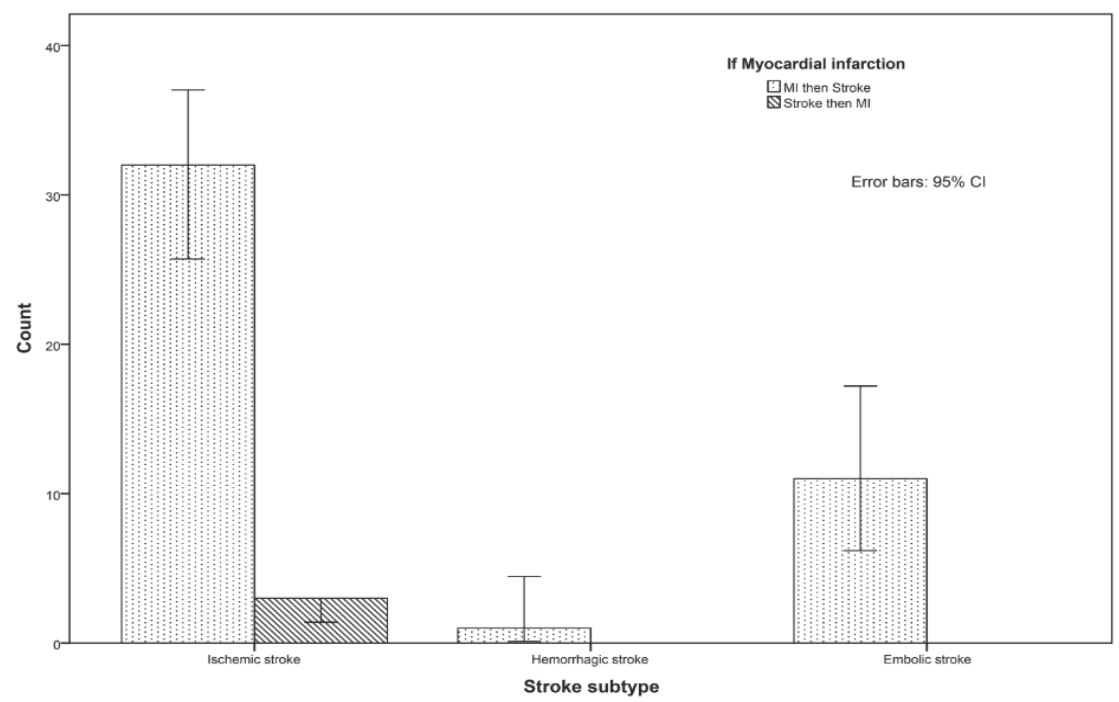

Figure 9. The association between myocardial infarction and stroke subtype patients with cerebrovascular accidents from Hail region in Saudi Arabia. [ischemic stroke: $68.09 \%$ MI then stroke and 6.38\% stroke then MI; hemorrhagic stroke: $2.19 \%$ MI then stroke and No stroke then MI; embolic stroke: $23.40 \%$ MI then stroke and No stroke then MI].

stroke patients were live. The overall status of stroke patients were shown $92.1 \%$ live and $7.9 \%$ were dead approximately during the period of 2016 to 2017 .

\section{Discussion}

Cerebrovascular disorders are common conditions leading to significant morbidity and mortality in the population. The generally recognized importance of cerebrovascular accidents (CVAs) as a cause of death has prompted the World Health Organization to sponsor centers in Europe, Asia, and Africa to study this problem [8]. Both CVA incidence and the mortality resulting from CVAs exhibit wide geographic variations, even within a single country [8-12], variations that have not yet been satisfactorily explained $[11,13]$.

The largest country in the Middle East is Saudi Arabia, occupies nearly four-fifths of the Arabian Peninsula with more than 28 million population. In Saudi Arabia, yearly $22 \%$ of deaths occurs by CVD [14]. But the current data on stroke in the Saudi Arabia are lacking [15]. The prevalence of stroke in Saudi Arabia is more intricate to estimate precisely. A prevalence of 178 out of 100,000 was reported in a community based survey from the Eastern region of Saudi $[16,17]$. Compare with Western countries, the Saudi Arabia has the lower rates in the incidence and prevalence of stroke.

In 2007 reported that a major percentage of the patients had not even heard the term "stroke" in the Gulf Cooperation Council (GCC) countries [18]. Obesity, smoking, sedentary lifestyle, etc are the risk factors for stroke. A study indicates in Saudi Arabia that prevalence of stroke among men than women [19]. In another study showed interviewed neurologists about their views on stroke, reports that acute stroke care in Saudi Arabia as inadequate [20].

In 2012 a study reported an alarming deficit in the level of stroke awareness in the Saudi population [21]. Stroke was more frequent in the sixth decade of life with male predominance in both Saudis and non-Saudis [22-24]. A study conducted in King Abdul Aziz University Hospital, Saudi arabia found that there was a high incidence of thrombotic stroke [25].

Across middle east, in Qatar the incidence rate for all strokes
63/100,000 population. But in Libya, the incidence rates for hemorrhagic stroke were 2.7/100,000. Saudi Arabia showed $1.9 / 100,000$ stroke incidence and 10.4/100,000 in Iran [26]. The study from Majmaah Province, Saudi Arabia indicates that noncardioembolic disease was the overwhelming stroke mechanism in this middle-eastern cohort with only $4 \%$ of patients have cardioembolic stroke, in comparison to roughly $20 \%$ reported in Western cohorts. Further, 59\% of the cohort had concurrence of hypertension and diabetes mellitus [27]. This indicates that the major risk factors for CVA are hypertension and diabetes mellitus showed in our study Figures 5 and 7.

The present study describes several notable findings. We observed that patients with CVA are not at an increased risk of stroke death, as compared to age, and sex. The prevalence of cerebrovascular accident (CVA) or stroke is increasing in frequency amongst the general population due to increased prevalence of diabetes and hypertension [28]. Stroke poses the risk of immediate mortality and long-term morbidity in the survivors with residual disability. The commonest underlying etiology of the CVA is vascular occlusion leading to infarction of brain [29]. Hemorrhagic stroke is less frequent than ischemic stroke and is due to aneurysmal rupture or malignant hypertension [30].

In general, elevated sugar (Figure 7), LDL-cholesterol, hypertension (Figure 5), and cigarette smoking (Figure 6) are strongly associated with an increased risk of myocardial infarction and CVA. The increasing incidence of hypertension with age had already been described before [31-37]. Hemorrhagic stroke is a rare but serious complication after an acute myocardial infarction (AMI). But the overall incidence of ischemic stroke within 5 months after acute myocardial infarction was $85.26 \%$ over the period September 2017-January 2018 in Hail region, Saudi Arabia. Ischemic stroke was more predominant compared with other subtypes. The hemorrhagic stroke and embolic stroke were shown an equal percentage (7.37\%) of CVA patients. Patients with hemorrhagic stroke have higher short-term mortality, but ischemic stroke patients have a significant long-term disability and morbidity [38]. 
Citation: Nawaf A, Hala A, Ahmed A, et al. Prevalence and prognosis of cerebrovascular accidents and its subtypes: A cross-sectional study in Hail region, Saudi Arabia. J Neurol Neurorehabil Res. 2019;4(1):1-8.

In the present study, the risk of CVA in patients with diabetes mellitus was $55.79 \%$ of ischemic stroke, $40 \%$ of hemorrhagic stroke and $7.37 \%$ of the CVA were embolic stroke. Although the incidence of CVA was higher in adult patients with ischemic stroke, we did not find a strong prediction from age and sex. We demonstrated that hypertension was the most significant predictor for CVA. Despite a wide variety of risk factors described for CVAs [39], hypertension is generally accepted as the principal predisposing factor [40]. From this study, several insights and recommendation for improving care extended to stroke patient can be derived.

\section{Conclusion}

In conclusion we demonstrated that diabetic and hypertensive patients have higher mortality after incident stroke, but sex and age was not an independent risk factor. Incidence of complicating hospitalizations with cerebrovascular accident continues to grow and is associated with increased mortality and adverse discharge. This highlights the need for early diagnosis, better risk stratification, and preparedness for need for complex long-term care in this vulnerable population.

\section{Acknowledgements}

We are grateful to Professor (Dr). Awdah Al-Hazimi, Dean of College of Medicine, University of Hail, for his encouragement and providing facilities. We render our heartfelt thanks to the CVA patients who permit to access their medical record for this study without any hesitation, we pray God for their relief from the disease.

\section{References}

1. Sims NR, Muyderman H. Mitochondria, oxidative metabolism and cell death in stroke. Biochim Biophys Acta. 2010;1802(1):80-9.

2. Shivaraju A, Yu C, Kattan MW, et al. Temporal trends in percutaneous coronary intervention-associated acute cerebrovascular accident (from the 1998 to 2008 Nationwide Inpatient Sample Database). Am J Cardiol. 2014;114:206-13.

3. Korn-Lubetzki I, Farkash R, Pachino RM, et al. Incidence and risk factors of cerebrovascular events following cardiac catheterization. J Am Heart Assoc. 2013;2:e000413.

4. Nichols J, Townsend N, Luengo-Fernandez R, et al. European cardiovascular disease statistics. Brussels, Sophia - Antipolis: European Heart Network and European Society of Cardiology. 2012.

5. Benamer HT, Grosset D. Stroke in Arab countries: A systematic literature review. J Neurol Sci. 2009;284(1-2):18-23.

6. O'Donnell MJ, Chin SL, Rangarajan S, et al. Global and regional effects of potentially modifiable risk factors associated with acute stroke in 32 countries (INTERSTROKE): A casecontrol study. Lancet. 2016;388(10046):761-75.

7. Al-Jadid MS, Robert AA. Determinants of length of stay in an inpatient stroke rehabilitation unit in Saudi Arabia. Saudi Med J. 2010;31(2):189-92.

8. World Health Organization. Cerebrovascular diseases: Prevention, treatment, and rehabilitation. WHO Technical Report Series, No. 469. Geneva. 1971.

9. Aho K, Harmsen P, Hatano S, et al. Cerebrovascular disease in the community: Results of a WHO collaborative study. Bull WHO. 1980;58(1):113-30.

10. Borhani NO. Changes and geographic distribution of mortality from cerebrovascular disease. Am J Public Health. 1965;55(5):673-681.

11. Marquardsen J. The epidemiology of cerebrovascular disease. Acta Neurol. 1978;57:57-75.

12. Richard J. Epidemiology of hypertension and stroke in Europe and the mediterranean countries. In: S. Hatano, I. Shigematsu, and T. Strasser (eds.). Hypertension and stroke control in the community: Proceedings of a WHO meeting held in Tokyo, 11-13 March 1974. World Health Organization, Geneva. 1976;pp:60-78.

13. Hatano S. Experiences with community strokes registers. In: S. Hatano, I. Shigematsu, and T. Strasser (eds.). Hypertension and stroke control in the community: Proceedings of a WHO meeting held in Tokyo, 11-13 March 1974. World Health Organization, Geneva. 1976;pp:117-28.

14. Robert A, Zamzami MM. Stroke in Saudi Arabia: A review of the recent literature. The Pan African Medical Journal. 2014; $17: 14$

15. Alahmari K. Prevalence of stroke in kingdom of Saudi Arabia - Through a physiotherapist diary. Mediterranean Journal of Social Sciences. 2016;7(1):228-33.

16. Al-Rajeh S, Awada A. Stroke in Saudi Arabia. Cerebrovasc Dis. 2002;13(1):3-8.

17. Awada A. Primary and secondary prevention of ischemic stroke. J Med Liban. 2011;59(4):213-9.

18. Kamran S, Bener AB, Deleu D, et al. The level of awareness of stroke risk factors and symptoms in the Gulf Cooperation Council countries: Gulf Cooperation Council stroke awareness study. Neuroepidemiology. 2007;29(3-4):235-42.

19. Yaqub BA, Shamena AR, Kolawole TM, et al. Cerebrovascular disease in Saudi Arabia. Stroke. 1991; 22(9):1173-6.

20. Al-Khathaami AM, Algahtani H, Alwabel A, et al. The status of acute stroke care in Saudi Arabia: An urgent call for action. Int J Stroke. 2011;6(1):75-6.

21. Al-Aqeel A, Al-Ammari B, Alsyefi N, et al. Stroke awareness in the Saudi Arabia Community: Prompt Public Health Measures Must Be Implement. 2012.

22. Akbar D, Mushtaq M, Quaboos S. Clinical profile of stroke at King Abdulaziz University hospital. Journal for Scientific Research. 2001;1:35- 38.

23. Al-Jishi AA, Mohan PK. Profile of stroke in Bahrain. Neurosiences. 2000;5:30-4.

24. Awada A, Rajeh S. The Saudi stroke data bank: Analysis of the first 1000 cases. Acta Neurol Scand. 1999;100(4):265-9.

25. Qari FA. Profile of stroke in a teaching university hospital in the western region. Saudi Med J. 2000;21(11):1030-3.

26. Tran J, Mirzaei M, Anderson L, et al. The epidemiology of stroke in the Middle East and North Africa. J Neurol Sci. 2010;295(1-2):38-40. 
27. Al-Harbi A, Shoamanesh A. Stroke subtypes and risk factors in Saudi Arabia. Neurology. 2016;86 (16):124.

28. Allen CL, Bayraktutan U. Risk factors for ischaemic stroke. Int J Stroke. 2008;3:105-16.

29. Stürmer T, Schlindwein G, Kleiser B, et al. Clinical diagnosis of ischemic versus hemorrhagic stroke: Applicability of existing scores in the emergency situation and proposal of a new score. Neuroepidemiology. 2002;21:8-17.

30. Sundquist K, Li X, Hemminki K. Familial risk of ischemic and hemorrhagic stroke: A large-scale study of the Swedish population. Stroke. 2006;37:1668-73.

31. Webb G. Treatment of coarctation and late complications in the adult. Semin Thorac Cardiovasc Surg. 2005;17:139e142.

32. Brown ML, Burkhart HM, Connolly HM, et al. Coarctation of the aorta: Lifelong surveillance is mandatory following surgical repair. J Am Coll Cardiol. 2013;62:1020e1025.

33. Roche SL, Silversides CK. Hypertension, obesity, and coronary artery disease in the survivors of congenital heart disease. Can J Cardiol. 2013;29:841e848.

34. O'Sullivan JJ, Derrick G, Darnell R. Prevalence of hypertension in children after early repair of coarctation of the aorta: A cohort study using casual and 24-hour blood pressure measurement. Heart. 2002;88:163e166.

35. Chiu HH, Chiu SN, Hu FC, et al. Late cardiovascular complications after surgical or balloon angioplasty of coarctation of aorta in an Asian cohort. Am J Cardiol. 2009; 104:1139e1144.

36. Hager A, Kanz S, Kaemmerer H, et al. Coarctation Longterm Assessment (COALA): Significance of arterial hypertension in a cohort of 404 patients up to 27 years after surgical repair of isolated coarctation of the aorta, even in the absence of restenosis and prosthetic material. J Thorac Cardiovasc Surg. 2007; 134:738e745.

37. Moltzer E, Mattace-Raso FU, Karamermer Y, et al. Comparison of candesartan vs. metoprolol for treatment of systemic hypertension after repaired aortic coarctation. Am J Cardiol. 2010;105:217e222.

38. Bamford J, Sandercock P, Dennis M, et al. Classification and natural history of clinically identifiable subtypes of cerebral infarction. Lancet. 1991;337:1521-6.

39. Neuman J, Mettinger KL, Soderstrom CE. Stroke in males before 55: A study of 206 patients. Acta Nemol scand. 1978;pp:57-222.

40. Svenius J, Pyr-Blalill K, Riekkinen PH, et al. The incidence of stroke in Kuopio area, Finland. Acta Neural Scand. 1980;62:193.

\section{*Correspondence to:}

Nawaf Alhazmi

Department of Internal Medicine,

College of Medicine,

University of Hail, Saudi Arabia

Tel : +966-503332081

E-mail : Nawaf415@hotmail.com 